\title{
Clinical Features and Risk Factors for Nasal Rosacea: A Hospital-Based Retrospective Study
}

\author{
Ai-ke Wu $\cdot$ Fang-fen Liu $\cdot$ Hong-fu Xie $\cdot$ Zhi-xiang Zhao • \\ Yan Tang · Ying-xue Huang · Dan Jian · Wei Shi · Ben Wang (D) • \\ Ji Li
}

Received: June 24, 2021 / Accepted: August 27, 2021 / Published online: September 4, 2021

(C) The Author(s) 2021

\section{ABSTRACT}

Introduction: At present, some studies have reported that nasal rosacea may be an independent disease, but phenotypic characteristics and risk factors for nasal rosacea remain unknown. This study aimed to clarify the clinical features and explore the risk factors for nasal rosacea.

Methods: A hospital-based retrospective study was conducted, including 1615 rosacea patients and 1501 healthy individuals. The patients were

Ai-ke Wu and Fang-fen Liu contribute equally to this work.

A. Wu $\cdot$ F. Liu $\cdot$ H. Xie - Z. Zhao $\cdot$ Y. Tang

Y. Huang · D. Jian · W. Shi · B. Wang $(\varangle) \cdot$ J. Li (ه)

Department of Dermatology, Xiangya Hospital,

Central South University, Xiangya Road, Kaifu

District, Changsha 410008, China

e-mail: wangben0804@163.comJ. Li

e-mail: liji_xy@csu.edu.cn

A. Wu · F. Liu $\cdot$ H. Xie - Z. Zhao · Y. Tang .

Y. Huang · D. Jian · W. Shi · B. Wang · J. Li

Key Laboratory of Aging Biology of Hunan Province,

Xiangya Hospital, Central South University,

Changsha, China

F. Liu $\cdot$ H. Xie $\cdot$ Z. Zhao · Y. Tang $\cdot$ Y. Huang .

D. Jian · W. Shi · B. Wang · J. Li

National Clinical Research Center for Geriatric

Disorders, Xiangya Hospital, Central South

University, Changsha 410008, China divided into three groups based on the involved areas of the lesions (non-nasal, intermediate and nasal rosacea group). Their demographic data and clinical features were obtained from patients' medical records, and risk factors of nasal rosacea were analyzed.

Results: There were 927 (57.4\%), 647 (40.1\%) and $41(2.5 \%)$ cases in the non-nasal, intermediate and nasal rosacea groups, respectively. Of 41 patients with nasal rosacea, all $(100.0 \%)$ had fixed erythema and 17 cases (41.5\%) had phymatous changes. Compared with control group, male gender (adjusted odds ratio $[\mathrm{aOR}]=2.39$, $95 \%$ confidence interval $[\mathrm{CI}]=1.14,4.99)$, obesity $(\mathrm{aOR}=3.19,95 \%$ CI $1.86,11.79)$ and alcohol use $(\mathrm{aOR}=1.58,95 \% \mathrm{CI} 1.22,5.40)$ were risk factors for nasal rosacea, but these three factors were not risk factors for non-nasal rosacea and intermediate rosacea groups. Among patients with nasal lesions (compared with patients without nasal phymatous changes), family history of rosacea was a risk factor $(\mathrm{aOR}=2.12,95 \% \mathrm{CI} 1.01,4.46)$ for nasal phymatous changes and Fitzpatrick IV skin type was a protective factor $(\mathrm{aOR}=0.49,95 \%$ CI 0.28 , $0.86)$.

Conclusion: Nasal rosacea has relatively specific clinical features and independent risk factors, suggesting that it may be a special type of rosacea.

Keywords: Phenotype; Phymatous change; Nasal rosacea; Rosacea; Risk factor 


\section{Key Summary Points}

Nasal rosacea refers to rosacea with nasal lesions, which has been thought to be an independent disease, but phenotypic characteristics and risk factors for nasal rosacea remain unkown

We clarified the clinical features and explored the risk factors for nasal rosacea, expecting nasal rosacea would be different from extra-nasal rosacea

After data collection and risk factor analysis, we were surprised to find that nasal rosacea has relatively specific clinical features and independent risk factors

On the basis of our study's results, we consider that nasal rosacea may be a special type of rosacea and need more attention

\section{INTRODUCTION}

Rosacea is a common chronic inflammatory skin condition involving mainly the convexities of the central face, including the cheeks, nose, chin and central forehead [1]. Rosacea is conventionally classified into four major subtypes: erythematotelangiectatic rosacea (ETR), papulopustular rosacea (PPR), phymatous rosacea (PhR) and ocular rosacea [2]. Currently, new classification favors a patient-tailored phenotype rather than classification based in subtypes; phenotypes in this case include flushing, fixed erythema, papules and pustules, telangiectasia, phymatous changes, burning and tingling, etc. [3-7]. To date, many risk factors for rosacea, such as family history, types of photosensitivity, alcohol consumption and others, have been reported [8-14].

In terms of primary areas, cheeks and nose are the most commonly affected areas [15]. It has been clinically observed that some patients tend to have nasal-confined lesions. Only one early-phase study with a small sample size has reported that localized nasal rosacea frequently presented with the ETR and PhR subtypes, which were different from those exhibited by extra-nasal rosacea, and hypothesized that nasal rosacea might be an independent condition [16]. However, this study did not provide a description of the phenotypic characteristics of rosacea. Moreover, our previous study also demonstrated that lesions in different facial areas in patients with rosacea showed significant differences in clinical features, and this was through a simply parallel comparison of rosacea in different facial areas [17]. Nevertheless, these two studies neither focused on risk factors of nasal rosacea nor paid attention to the progress and related factors of nasal phymatous changes.

Based on the current stage of research, many issues still need to be resolved. Some of these unanswered questions include: What are the phenotypic characteristics of nasal rosacea? Are these characteristics distinct from those of rosacea in other facial areas? What are the unique factors of nasal rosacea? Could nasal rosacea really be considered a special type of rosacea?

The current study was mainly designed to describe the phenotypic characteristics of nasal rosacea in detail and analyze the possible risk factors as well as provide clinical data on nasal rosacea to promote precise treatment and patient education and lay the foundations for further research into the mechanisms of this special type of rosacea.

\section{METHODS}

\section{Study Design and Participants}

This study was a hospital-based cross-sectional study, including 1615 patients and 1501 healthy individuals. All 1615 patients were diagnosed with rosacea according to the criterion of the aligned 2019 ROSacea COnsensus (ROSCO) and 2017 National Rosacea Society phenotype recommendations $[4,6]$. These patients were admitted to the Xiangya Hospital from July 2017 to March 2019 and enrolled in the study 
consecutively. Exclusion criteria comprised symptoms and diseases interfering with the evaluation, including erosion, steroid use, severe bacterial or fungal infection, other skin disorders, pediatric diseases, pregnancy and history of systematic diseases, whereas patients with acne were not excluded. Moreover, the patients needed to be newly diagnosed patients and not to have been treated recently. Therefore, patients who had used topical drugs within 2 weeks, used tetracycline oral drugs or photoelectric therapy within 1 month or used isotretinoin within 6 months, at either our hospital or another institution, were excluded from the study. Healthy individuals were included as healthy control group, who were physical examination personnel without history of skin diseases or other systematic diseases in the physical examination center at the same period. The study was performed in accordance with the ethical standards as laid down in the 1964 Declaration of Helsinki and its later amendments or comparable ethical standards. The detailed protocol of this study was approved by the Institutional Review Board of Xiangya hospital, Central South University (Ethical Application Ref: 201611608). Informed written consents were obtained from every participant.

\section{Data Collection}

Demographic and clinical data were gathered from patients' medical records, including age, sex, body mass index (BMI), marital status, occupation, years of education, personal monthly income, skin type, Fitzpatrick skin type (Fitzpatrick III-sometimes burn, usually tan; Fitzpatrick IV—never burn, always tan), smoking status and alcohol use. Among these variables, age was recorded in four categories: < 24 years old, 25-34 years old, 35-44 years old and $>44$ years old; body mass index, which was calculated as weight in kilograms divided by height in meters squared, was classified into three categories: underweight (BMI $<18.5 \mathrm{~kg} /$ $\left.\mathrm{m}^{2}\right)$, normal $\left(18.5 \mathrm{~kg} / \mathrm{m}^{2} \leq \mathrm{BMI}<24 \mathrm{~kg} / \mathrm{m}^{2}\right)$ and overweight or obese $\left(\mathrm{BMI} \geq 24 \mathrm{~kg} / \mathrm{m}^{2}\right)$; years of education was recorded in two categories: < 12 years or 12 years; > 12 years. Personal monthly income was recorded in three levels: high, middle and low income. Occupation was recorded in two categories: physical and non-physical labor. The clinical phenotypic features collected included flushing, persistent erythema, telangiectasia, papules/pustules, phymatous changes and ocular manifestations, according to the global scale provided by the 2017 ROSCO panel [6]. Moreover, patients' clinical data on whether acne and dermagraphia existed were collected from patients via dermatologists' observation and inquiry. Among them, the record of dermagraphia required dermatologists to perform a skin scratch test. (The physician uses a device such as a broken cotton swab to draw a line slowly on the flexion of the patient's forearm. If there is redness, edema, swelling or exudation at the scratched skin, and it does not disappear for $>$ $20 \mathrm{~min}$, it can be judged positive). In this study, patients were classified into three groups: (1) nasal rosacea group (rosacea lesions limited to the nose); (2) intermediate rosacea group (rosacea lesions with both nasal and extra-nasal areas); (3) non-nasal rosacea group (rosacea lesions without nasal involvement).

\section{Statistical Analysis}

Patient clinico-demographic characteristics were compared among all groups using the Pearson chi-square test for categorical variables and Kruskal-Wallis test for ranked variables. All categorical variables and ranked variables were represented by values and proportions. Multivariate logistic regression analysis was performed, and odds ratios (OR) and 95\% confidence intervals (CI) were calculated. The adjusted OR (aOR) was also calculated in the regression model. All reported $P$-values are two sided with $\alpha=0.05$. All statistical analyses were performed using SPSS version 25.0 (SPSS Inc., Chicago, IL, USA). 


\section{RESULTS}

\section{Demographic Characteristics}

The cohort study consisted of 1615 patients with rosacea and 1501 healthy individuals. Of these patients, 927 (57.4\%) had rosacea without nasal involvement (non-nasal rosacea group), 647 (40.1\%) had rosacea with nasal lesions (intermediate rosacea group), and 41 (2.5\%) had rosacea with nose-confined lesions (nasal rosacea group). Generally, there were significant differences between the control and total rosacea groups related to all characteristics studied, except for smoking status $(P=0.155)$, age $(P<0.001), \quad$ gender $\quad(P<0.001), \quad$ BMI $(P=0.004)$, marital status $(P<0.001)$, occupation $(P<0.001)$, number of years of education $(P<0.001)$, personal monthly income $(P=0.033)$, Fitzpatrick skin type $(P<0.001)$ and alcohol consumption $(P=0.01)$. The proportion of men in the nasal rosacea group was higher than that in the intermediate rosacea (39.0\% vs. $13.1 \%, P<0.001)$ and the non-nasal rosacea groups (39.0\% vs. $5.7 \%, P<0.001)$; all other characteristics were similar in each patient group (Table 1).

\section{Clinical Features of the Nasal Rosacea Group}

Since the diagnosis and classification of rosacea have evolved from the subtype to the phenotype approach, unlike previous studies, our study analyzed clinical features based on patient phenotypes rather than conventional subtypes. Of 41 patients in the nasal rosacea group, all (100.0\%) had erythema (Fig. 1a), 17 (41.5\%) had phymatous changes (Fig. 1b), 12 (29.3\%) had flushing, $11(26.8 \%)$ had papules and pustules, $6(14.6 \%)$ had burning and tingling sensations, $4(9.8 \%)$ had telangiectasia, and 1 $(2.4 \%)$ had rosacea combined with ocular manifestations. In addition, the phymatous changes seen in the 17 patients were all accompanied by erythema (Fig. 1b). The majority of patients developed nasal hyperplasia within 3-5 years, and a few patients could develop it within 10-20 years, reflecting the progression from onset to the phymatous changes (Fig. 2).

\section{Risk Factor Analysis}

\section{Risk Factors for Distinct Groups of Rosacea}

Compared with the control group, male gender $(\mathrm{aOR}=0.47,95 \%$ CI $0.35,0.63)$ and Fitzpatrick skin type IV $(\mathrm{aOR}=0.58 ; 95 \%$ CI $0.48,0.71)$ were found to be protective factors for the total rosacea group. The result of comparing the control group with the non-nasal group was consistent with that of the total rosacea group (male gender: $\mathrm{aOR}=0.27,95 \%$ CI $0.18,0.41$; Fitzpatrick skin type: IV aOR $=0.60,95 \%$ CI $0.48,0.75)$. However, only skin type IV had protective effects on patients in the intermediate rosacea group $(\mathrm{aOR}=0.58 ; 95 \%$ CI 0.45 , $0.75)$, and male gender was no longer a protective factor $(\mathrm{aOR}=0.74,95 \%$ CI $0.52,1.07)$. Different from other groups, when comparing the control group with the nasal rosacea group, male gender $(\mathrm{aOR}=2.39 ; 95 \%$ CI 1.14, 4.99), being overweight or obese $(\mathrm{aOR}=3.19 ; 95 \% \mathrm{CI}$ $1.86,11.7)$ and consumption of alcohol $(\mathrm{aOR}=1.58 ; 95 \% \mathrm{CI} 1.22,5.40)$ were risk factors for the nasal rosacea group, and skin type IV was not found to be associated with nasal rosacea $(\mathrm{aOR}=0.79,95 \%$ CI $0.38,1.67)$ (Table 2$)$.

\section{Risk Factors Associated with Nasal Phymatous Changes}

A total of 647 cases of intermediate rosacea and 41 cases of nasal rosacea were mixed, meaning both were characterized by patients with nasal lesions. Among 688 observed patients, there were $86(12.5 \%)$ patients with nasal phymatous changes and 602 (87.5\%) without these changes. Compared with patients without phymatous changes, Fitzpatrick skin type IV was a protective factor for nasal phymatous changes $(\mathrm{aOR}=0.49 ; 95 \%$ CI $0.28,0.86 ; P=0.011$ for trend), and family history of rosacea was a risk factor for developing nasal phymatous changes $(\mathrm{aOR}=2.12 ; 95 \%$ CI 1.01, 4.46; $P=0.049$ for trend). In contrast to risk factors for nasal rosacea, there were no differences between the nasal phymatous and control groups (patients without phymatous changes) with aspects to 
Table 1 Distributions of selected characteristics related to total rosacea, non-nasal rosacea, intermediate rosacea, nasal rosacea and controls

\begin{tabular}{|c|c|c|c|c|c|c|c|c|c|c|c|c|}
\hline \multirow[t]{2}{*}{ Characteristics } & \multicolumn{2}{|c|}{ Controls } & \multicolumn{2}{|c|}{$\begin{array}{l}\text { Total } \\
\text { rosacea }\end{array}$} & \multirow[t]{2}{*}{$P$} & \multicolumn{2}{|c|}{$\begin{array}{l}\text { Non-nasal } \\
\text { rosacea }\end{array}$} & \multicolumn{2}{|c|}{$\begin{array}{l}\text { Intermediate } \\
\text { rosacea }\end{array}$} & \multicolumn{2}{|c|}{$\begin{array}{l}\text { Nasal } \\
\text { rosacea }\end{array}$} & \multirow[t]{2}{*}{$P$} \\
\hline & $\begin{array}{l}N= \\
1501\end{array}$ & $\%$ & $\begin{array}{l}N= \\
1615\end{array}$ & $\%$ & & $\begin{array}{l}N= \\
927\end{array}$ & $\%$ & $\begin{array}{l}= \\
647\end{array}$ & $\%$ & $\begin{array}{l}N= \\
41\end{array}$ & $\%$ & \\
\hline Age (years) & & & & & $<0.001$ & & & & & & & 0.492 \\
\hline$<25$ & 385 & 25.6 & 539 & 33.4 & & 302 & 32.6 & 219 & 33.8 & 18 & 43.9 & \\
\hline $25-34$ & 784 & 52.2 & 433 & 26.8 & & 255 & 27.5 & 170 & 26.3 & 8 & 19.5 & \\
\hline $35-44$ & 205 & 13.7 & 343 & 21.2 & & 203 & 21.9 & 135 & 20.9 & 5 & 12.2 & \\
\hline$>44$ & 127 & 8.5 & 300 & 18.6 & & 167 & 18.0 & 123 & 19.0 & 10 & 24.4 & \\
\hline Gender & & & & & $<0.001$ & & & & & & & $<0.001$ \\
\hline Male & 279 & 18.6 & 154 & 9.5 & & 53 & 5.7 & 85 & 13.1 & 16 & 39.0 & \\
\hline Female & 1222 & 81.4 & 1461 & 90.5 & & 874 & 94.3 & 562 & 86.9 & 25 & 61.0 & \\
\hline BMI & & & & & 0.004 & & & & & & & 0.584 \\
\hline Underweight & 288 & 19.2 & 312 & 19.3 & & 178 & 19.2 & 127 & 19.6 & 7 & 17.1 & \\
\hline Normal & 1067 & 71.1 & 1085 & 67.2 & & 633 & 68.3 & 426 & 65.8 & 26 & 63.4 & \\
\hline Overweight or obese & 146 & 9.7 & 218 & 13.5 & & 116 & 12.5 & 94 & 14.5 & 8 & 19.5 & \\
\hline Marital status & & & & & $<0.001$ & & & & & & & 0.497 \\
\hline Unmarried & 820 & 54.6 & 416 & 25.8 & & 247 & 26.6 & 157 & 24.3 & 12 & 29.3 & \\
\hline Married & 681 & 45.4 & 1199 & 74.2 & & 680 & 73.4 & 490 & 75.7 & 29 & 70.7 & \\
\hline Occupation & & & & & $<0.001$ & & & & & & & 0.741 \\
\hline Physical labor & 718 & 47.8 & 689 & 42.7 & & 388 & 41.9 & 282 & 43.6 & 19 & 46.3 & \\
\hline Non-physical labor & 783 & 52.2 & 926 & 57.3 & & 539 & 58.1 & 365 & 56.4 & 22 & 53.7 & \\
\hline Years of education & & & & & $<0.001$ & & & & & & & 0.121 \\
\hline$\leq 12$ years & 296 & 19.7 & 937 & 58.0 & & 539 & 58.1 & 368 & 56.9 & 30 & 73.2 & \\
\hline$>12$ years & 1205 & 80.3 & 678 & 42.0 & & 388 & 41.9 & 279 & 43.1 & 11 & 26.8 & \\
\hline Personal monthly income & & & & & 0.033 & & & & & & & 0.195 \\
\hline High income & 414 & 27.6 & 499 & 30.9 & & 296 & 31.9 & 191 & 29.5 & 12 & 29.3 & \\
\hline Middle income & 635 & 42.3 & 690 & 42.7 & & 400 & 43.1 & 268 & 41.4 & 22 & 53.7 & \\
\hline Low income & 452 & 30.1 & 426 & 26.4 & & 231 & 24.9 & 188 & 29.1 & 7 & 17.1 & \\
\hline Fitzpatrick skin type & & & & & $<0.001$ & & & & & & & 0.230 \\
\hline III type & 999 & 66.6 & 1110 & 68.7 & & 627 & 67.6 & 458 & 70.8 & 25 & 61.0 & \\
\hline IV type & 502 & 33.4 & 505 & 31.3 & & 300 & 32.4 & 189 & 29.2 & 16 & 39.0 & \\
\hline Smoking & & & & & 0.155 & & & & & & & 0.589 \\
\hline
\end{tabular}


Table 1 continued

\begin{tabular}{|c|c|c|c|c|c|c|c|c|c|c|c|c|}
\hline \multirow[t]{2}{*}{ Characteristics } & \multicolumn{2}{|c|}{ Controls } & \multicolumn{2}{|c|}{ Total rosacea } & \multirow[t]{2}{*}{$P$} & \multicolumn{2}{|c|}{ Non-nasal rosacea } & \multicolumn{2}{|c|}{ Intermediate rosacea } & \multicolumn{2}{|c|}{ Nasal rosacea } & \multirow[t]{2}{*}{$P$} \\
\hline & $\begin{array}{l}N= \\
1501\end{array}$ & $\%$ & $\begin{array}{l}N= \\
1615\end{array}$ & $\%$ & & $\begin{array}{l}N= \\
927\end{array}$ & $\%$ & $\begin{array}{l}N= \\
647\end{array}$ & $\%$ & $\begin{array}{l}N= \\
41\end{array}$ & $\%$ & \\
\hline No & 88 & 5.9 & 115 & 7.1 & & 60 & 6.5 & 52 & 8.0 & 3 & 7.3 & \\
\hline Yes & 1413 & 94.1 & 1500 & 92.9 & & 867 & 93.5 & 595 & 92.0 & 38 & 92.7 & \\
\hline Alcohol use & & & & & 0.01 & & & & & & & 0.493 \\
\hline No & 208 & 13.9 & 128 & 7.9 & & 72 & 7.8 & 51 & 7.9 & 5 & 12.2 & \\
\hline Yes & 1293 & 86.1 & 1487 & 92.1 & & 855 & 92.2 & 596 & 92.1 & 36 & 87.8 & \\
\hline
\end{tabular}
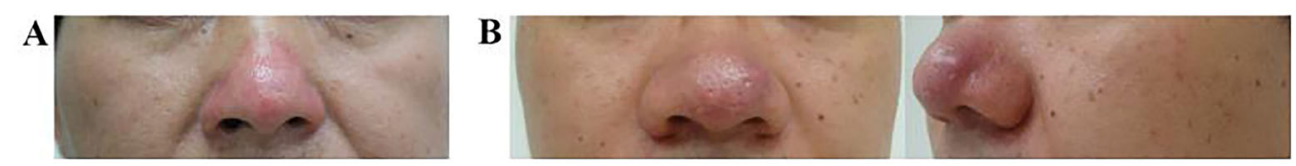

Fig. 1 A Male, 54 years old, erythema was found only on the nose. B Male, 55 years old, erythema and phymatous changes were found with papules and pustules on the nose

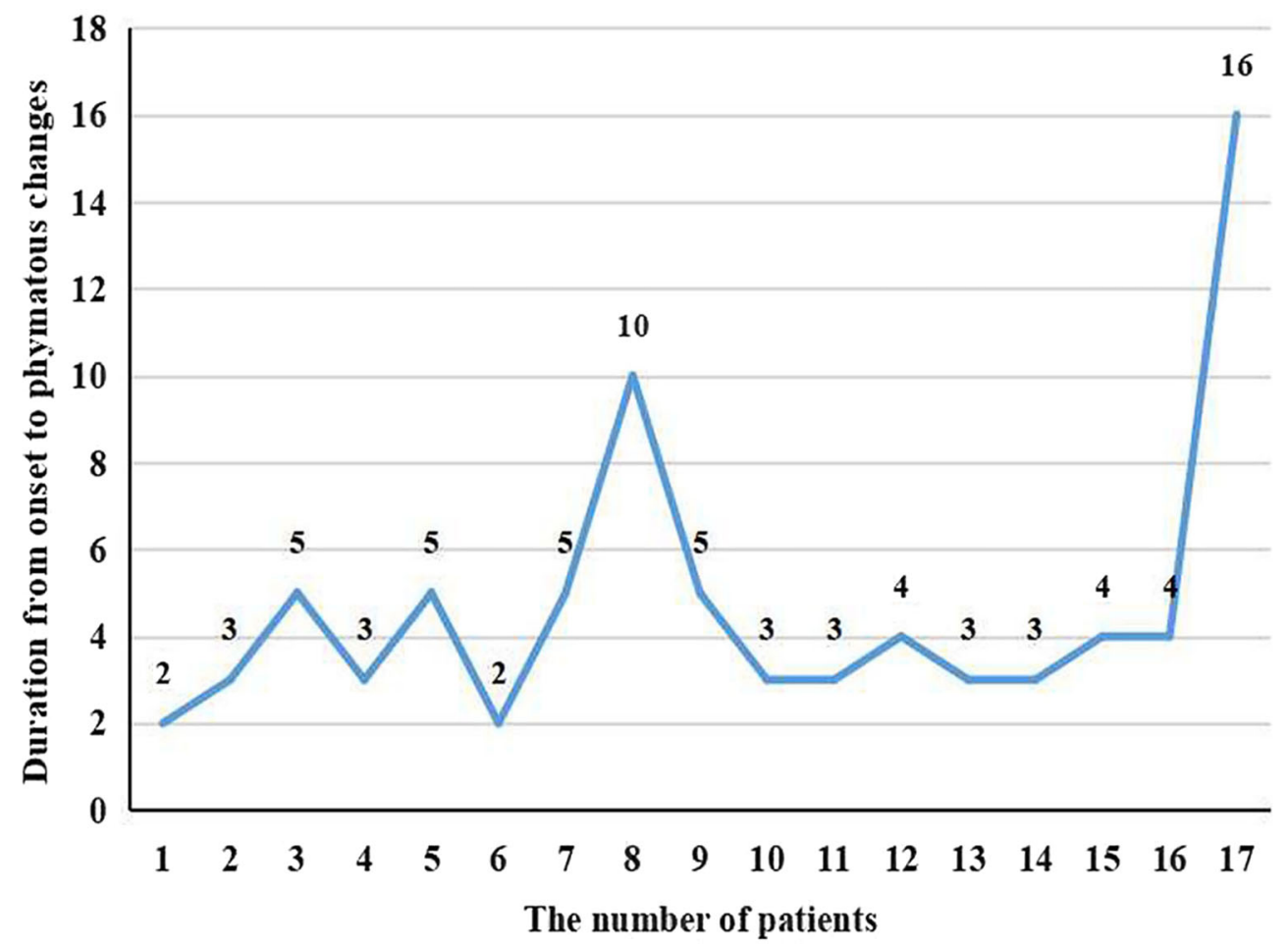

Fig. 2 Most patients developed nasal hyperplasia within 3-5 years, and for a few patients this was as long as 10-20 years 
Table 2 Odds ratios of non-nasal, intermediate and nasal rosacea compared with controls

\begin{tabular}{|c|c|c|c|c|}
\hline Characteristics & $\begin{array}{l}\text { Total rosacea } \\
\text { OR }_{\text {adj }}(95 \% \mathrm{CI})\end{array}$ & $\begin{array}{l}\text { Non-nasal rosacea } \\
\text { OR }_{\text {adj }}(95 \% \mathrm{CI})\end{array}$ & $\begin{array}{l}\text { Intermediate rosacea } \\
\mathrm{OR}_{\text {adj }}(95 \% \mathrm{CI})\end{array}$ & $\begin{array}{l}\text { Nasal rosacea } \\
\text { OR }_{\text {adj }}(95 \% \mathrm{CI})\end{array}$ \\
\hline \multicolumn{5}{|l|}{ Gender } \\
\hline Female & 1.00 & 1.00 & 1.00 & 1.00 \\
\hline Male & $0.47(0.35,0.63)$ & $0.27(0.18,0.41)$ & $0.74(0.52,1.07)$ & $2.39(1.14,4.99)$ \\
\hline \multicolumn{5}{|l|}{ BMI } \\
\hline Underweight & 1.00 & 1.00 & 1.00 & 1.00 \\
\hline Normal & $0.88(0.68,1.12)$ & $0.86(0.65,1.15)$ & $0.77(0.57,1.06)$ & $1.19(0.40,3.16)$ \\
\hline Overweight or obese & $1.34(0.92,1.93)$ & $1.37(0.90,2.09)$ & $1.39(0.89,1.77)$ & $3.19(1.86,11.79)$ \\
\hline \multicolumn{5}{|l|}{ Fitzpatrick skin type } \\
\hline III type & 1.00 & 1.00 & 1.00 & 1.00 \\
\hline IV type & $0.58(0.48,0.71)$ & $0.60(0.48,0.75)$ & $0.58(0.45,0.75)$ & $0.79(0.38,1.67)$ \\
\hline \multicolumn{5}{|l|}{ Smoking } \\
\hline No & 1.00 & 1.00 & 1.00 & 1.00 \\
\hline Yes & $1.12(0.69,1.80)$ & $1.56(0.91,2.68)$ & $0.97(0.54,1.74)$ & $0.23(0.03,2.11)$ \\
\hline \multicolumn{5}{|l|}{ Alcohol use } \\
\hline No & 1.00 & 1.00 & 1.00 & 1.00 \\
\hline Yes & $1.31(0.82,2.09)$ & $1.40(0.83,2.33)$ & $1.06(0.58,1.93)$ & $1.58(1.22,5.40)$ \\
\hline
\end{tabular}

$O R_{a d j}$ Adjusted by age, marital status, occupation, education and income vs. control group

The symbol [bold] represents the data has statistical significance

male gender $(\mathrm{aOR}=1.42$, 95\% CI $0.73,2.80)$, obesity $(\mathrm{aOR}=1.53,95 \%$ CI $0.64,3.65)$ and alcohol consumption $(\mathrm{aOR}=1.30,95 \% \mathrm{CI} 0.41$, 4.12) (Table 3).

\section{DISCUSSION}

Previous studies have evaluated the clinical features of nasal rosacea and progression between distinct subtypes [15-18], and only few studies have described the clinical features of nasal rosacea based on patients' phenotypic characteristics. Furthermore, no research has systematically analyzed the demographic characteristics and risk factors of nasal rosacea.

In our study, we applied a phenotypic approach to patients and demonstrated the clinical features of 41 patients with nasal rosacea. We found that all of them had fixed erythema and nearly half of them had nasal phymatous changes, while flushing and papulopustular conditions were phenotypes not commonly observed. Meanwhile, we found that the incidence rate of phymatous changes on the cheeks was markedly lower than that on the nose (only approximately 4.6\%; data not shown). This difference might arise from the divergent distribution of differentially functional fibroblast subpopulations in these two facial areas; detailed explanation in this regard requires further relevant studies [19]. Moreover, we observed that only a few patients had selfreported symptoms, such as burning and tingling sensations. Conversely, in our previous study, self-reported symptoms on the cheeks were more common [17]. Thus, taken together, we can conclude that the clinical features of nasal rosacea are indeed different from those of rosacea in other facial areas. 
Table 3 Factors associated with phymatous change among patients with nasal lesions

\begin{tabular}{|c|c|c|c|c|c|}
\hline \multirow[t]{2}{*}{ Related factors } & \multirow{2}{*}{$\begin{array}{l}\text { Observed population } \\
N=688\end{array}$} & \multicolumn{2}{|c|}{ Nasal phymatous } & \multirow[t]{2}{*}{$\mathrm{OR}_{\mathrm{adj}}(95 \% \mathrm{CI})$} & \multirow[t]{2}{*}{$P$} \\
\hline & & $N=86$ & $\%$ & & \\
\hline Gender & & & & & 0.306 \\
\hline Female & 587 & 70 & 11.9 & 1.00 & \\
\hline Male & 101 & 16 & 15.8 & $1.42(0.73,2.80)$ & \\
\hline BMI & & & & & 0.548 \\
\hline Underweight & 134 & 14 & 10.4 & 1.00 & \\
\hline Normal & 452 & 58 & 12.8 & $1.38(0.71,2.70)$ & \\
\hline Overweight or obese & 102 & 14 & 13.7 & $1.53(0.64,3.65)$ & \\
\hline Fitzpatrick skin type & & & & & 0.011 \\
\hline III type & 483 & 67 & 13.9 & 1.00 & \\
\hline IV type & 205 & 19 & 9.3 & $0.49(0.28,0.86)$ & \\
\hline Smoking & & & & & 0.548 \\
\hline No & 633 & 80 & 12.6 & 1.00 & \\
\hline Yes & 55 & 6 & 10.9 & $0.68(0.19,2.45)$ & \\
\hline Alcohol use & & & & & 0.656 \\
\hline No & 635 & 79 & 12.4 & 1.00 & \\
\hline Yes & 53 & 7 & 13.2 & $1.30(0.41,4.12)$ & \\
\hline Family history of rosacea & & & & & 0.049 \\
\hline No & 638 & 75 & 11.8 & 1.00 & \\
\hline Yes & 50 & 11 & 22.0 & $2.12(1.01,4.46)$ & \\
\hline Acne & & & & & 0.553 \\
\hline No & 463 & 60 & 13.0 & 1.00 & \\
\hline Yes & 225 & 26 & 11.6 & $0.88(0.51,1.49)$ & \\
\hline Dermagraphia & & & & & 0.214 \\
\hline No & 497 & 58 & 11.7 & 1.00 & \\
\hline Yes & 190 & 28 & 14.7 & $1.37(0.82,2.28)$ & \\
\hline Aczema & & & & & 0.605 \\
\hline No & 616 & 75 & 12.2 & 1.00 & \\
\hline Yes & 71 & 11 & 15.5 & $1.21(0.59,2.50)$ & \\
\hline
\end{tabular}

$O R_{a d j}$ Adjusted by age, duration, marital status, occupation, education and income

The symbol [bold] represents the data has statistical significance 
Since nasal rosacea has relatively specific clinical features and its pathogenesis is unclear, we investigated risk factors for nasal rosacea, hoping to provide some clinical clues regarding its etiology. We have concluded that male gender and Fitzpatrick IV skin type are protective factors for all rosacea and non-nasal rosacea groups; furthermore, Fitzpatrick skin type IV remains a protective factor for the intermediate rosacea group, further confirming the result of our previous studies [20, 21]. Conversely, Fitzpatrick skin type IV was no longer found to be a protective factor for nasal rosacea, and male gender, being overweight or obese, and consumption of alcohol were found to be risk factors instead. Our results provide new risk factors for nasal rosacea. Current findings show that hormones could affect rosacea via angiectasis and inflammatory cell infiltration, and men usually secrete more potent androgens than women after attainment of puberty $[22,23]$. As for obese individuals, adipose tissue can also synthesize androgen, promoting hyperandrogenism in these individuals; moreover, these individuals may have more sebaceous glands on their noses $[13,24]$. More importantly, there are more androgen receptors on the nose than cheeks [25]. These aforementioned findings might be the reasons why males and obese individuals are more prone to developing nasal rosacea, but detailed mechanistic studies are required in this regard. Alcohol consumption can stimulate vasodilation and aggravate persistent erythema, thus playing a role in the chronic vascular inflammation necessary to cause erythema on the nose $[26,27]$. Therefore, based on the risk factors mentioned herein, patients should be actively encouraged to avoid alcohol consumption and maintain a healthy weight to prevent nasal rosacea. According to our findings, nasal rosacea has specific clinical features and risk factors; thus, we hold the view that nasal rosacea might be considered a special type of rosacea, and future investigations on its pathogenic mechanisms are required.

In terms of nasal phymatous changes, our study found that Fitzpatrick skin type IV had a protective effect, while family history of rosacea was a risk factor. There are some conflicting reports on risk factors for phymatous changes on the nose (rhinophyma) $[9,10,28,29]$. A previous study showed no association between alcohol consumption and rhinophyma, while a recent case-control study revealed a highly significant association between the two $[10,29]$. In our study, alcohol consumption showed no association with nasal phymatous changes. Alcohol consumption in China is lower than in Western countries, especially among women. Furthermore, the types of alcohol also vary among different countries and should be taken into account. Moreover, consistent with a previous study [10], family history of rosacea was found to be a risk factor for nasal phymatous changes, probably because some specific genes contribute to nasal phymatous changes. Further studies on related genetic mechanisms are required. Additionally, we observed that all nasal phymatous changes occurred because of fixed erythema. Therefore, in clinical practice, more attention should be paid to the family histories of patients with rosacea and nasal fixed erythema; if family history of disease is noted, these patients should be treated promptly to avoid serious damage in the future, such as nasal hypertrophy.

\section{Limitations}

However, our study had several limitations. First, the clinical data we used were from our hospital, which covers only the South Central Chinese population. Therefore, it cannot be representative of the national population. Second, potential recall bias may exist during clinical data collection. Third, this was a retrospective study and thus could suggest only correlation rather than causation. Therefore, the above-mentioned findings still require further prospective and comprehensive investigation in a larger population.

\section{CONCLUSION}

In conclusion, we clarified the demographic and clinical features of patients with nasal rosacea and investigated the potential risk factors for nasal rosacea. We found that nasal rosacea has specific clinical features and 
independent risk factors, supporting the hypothesis that it may be a special type of rosacea. Even though the results of this study require further elucidation, they may provide comprehensive clinical data on nasal rosacea to enable further research and provide practical recommendations to prevent risk factors in patients.

\section{ACKNOWLEDGEMENTS}

We thank all of the participants involved in this study. Their contributions are gratefully acknowledged.

Funding. This work was primarily supported by National Natural Science Foundation of China (nos. 81773351, 81974480) and Cultivation Project of the National Natural Science Foundation Major Research Program (no. 91749114). The journal's Rapid Service Fee will be supported by National Natural Science Foundation of China (no. 81974480).

Authorship. All named authors meet the International Committee of Medical Journal Editors (ICMJE) criteria for authorship for this article, take responsibility for the integrity of the work as a whole, and have given their approval for this version to be published.

Author Contributions. Study conception and design were performed by Ben Wang and Ji Li. Material preparation, data collection and analysis were performed by Hong-fu Xie Zhixiang Zhao, Fang-fen Liu, Yan Tang, Ying-xue Huang, Dan Jian and Wei Shi. The first draft of the manuscript was written by Ai-ke Wu and Fang-fen Liu, and all authors commented on previous versions of the manuscript. All authors read and approved the final manuscript.

Disclosures. Ai-ke Wu, Fang-fen Liu, Hongfu Xie, Zhi-xiang Zhao, Yan Tang, Ying-xue Huang, Dan Jian, Wei Shi, Ben Wang and Ji Li have nothing to disclose.

Compliance with Ethics Guidelines. The detailed protocol of this study was approved by the Institutional Review Board of Xiangya Hospital, Central South University (Ethical Application Ref: 201611608). The study was performed in accordance with the ethical standards as laid down in the 1964 Declaration of Helsinki and its later amendments or comparable ethical standards. Written informed consents have been obtained from every participant.

Data Availability. All data generated or analyzed during this study are included in this published article.

Open Access. This article is licensed under a Creative Commons Attribution-NonCommercial 4.0 International License, which permits any non-commercial use, sharing, adaptation, distribution and reproduction in any medium or format, as long as you give appropriate credit to the original author(s) and the source, provide a link to the Creative Commons licence, and indicate if changes were made. The images or other third party material in this article are included in the article's Creative Commons licence, unless indicated otherwise in a credit line to the material. If material is not included in the article's Creative Commons licence and your intended use is not permitted by statutory regulation or exceeds the permitted use, you will need to obtain permission directly from the copyright holder. To view a copy of this licence, visit http:// creativecommons.org/licenses/by-nc/4.0/.

\section{REFERENCES}

1. Korting HC, Schollmann C. Current topical and systemic approaches to treatment of rosacea. J Eur Acad Dermatol Venereol. 2009;23:876-82.

2. Crawford GH, Pelle MT, James WD. Rosacea: I. Etiology, pathogenesis, and subtype classification. J Am Acad Dermatol. 2004;51:327-41 (Quiz 42-4).

3. Gallo RL, Granstein RD, Kang S, et al. Standard classification and pathophysiology of rosacea: the 2017 update by the National Rosacea Society Expert Committee. J Am Acad Dermatol. 2018;78:148-55. 
4. Schaller M, Almeida LMC, Bewley A, et al. Recommendations for rosacea diagnosis, classification and management: update from the global ROSacea COnsensus 2019 panel. Br J Dermatol. 2020;182: 1269-76.

5. Tan J, Berg M, Gallo RL, Del Rosso JQ. Applying the phenotype approach for rosacea to practice and research. Br J Dermatol. 2018;179:741-6.

6. Tan J, Almeida LM, Bewley A, et al. Updating the diagnosis, classification and assessment of rosacea: recommendations from the global ROSacea COnsensus (ROSCO) panel. $\mathrm{Br} \mathrm{J}$ Dermatol. 2017; 176:431-8.

7. Tan J, Steinhoff M, Berg M, et al. Shortcomings in rosacea diagnosis and classification. Br J Dermatol. 2017;176:197-9.

8. Abram K, Silm H, Maaroos HI, Oona M. Risk factors associated with rosacea. J Eur Acad Dermatol Venereol. 2010;24:565-71.

9. Alinia H, Tuchayi SM, Patel NU, et al. Rosacea triggers: alcohol and smoking. Dermatol Clin. 2018;36:123-6.

10. Second J, Severac F, Paix A, Cribier B. Rhinophyma is associated with alcohol intake. J Am Acad Dermatol. 2019;81:249-50.

11. Yuan X, Huang X, Wang B, et al. Relationship between rosacea and dietary factors: a multicenter retrospective case-control survey. J Dermatol. 2019;46:219-25.

12. Li S, Cho E, Drucker AM, Qureshi AA, Li WQ. Cigarette smoking and risk of incident Rosacea in women. Am J Epidemiol. 2017;186:38-45.

13. Li S, Cho E, Drucker AM, Qureshi AA, Li WQ. Obesity and risk for incident rosacea in US women. J Am Acad Dermatol. 2017;77:1083-7.

14. Li S, Chen ML, Drucker AM, et al. Association of caffeine intake and caffeinated coffee consumption with risk of incident rosacea in women. JAMA Dermatol. 2018;154:1394-400.

15. Tan J, Blume-Peytavi U, Ortonne JP, et al. An observational cross-sectional survey of rosacea: clinical associations and progression between subtypes. Br J Dermatol. 2013;169:555-62.

16. Lee WJ, Jung JM, Won KH, et al. Clinical evaluation of 368 patients with nasal rosacea: subtype classification and grading of nasal rosacea. Dermatology. 2015;230:177-83.
17. Xie HF, Huang YX, He L, et al. An observational descriptive survey of rosacea in the Chinese population: clinical features based on the affected locations. PeerJ. 2017;5: e3527.

18. Lee WJ, Lee YJ, Won CH, Chang SE, Choi JH, Lee MW. Clinical evaluation of 30 patients with localized nasal rosacea. J Dermatol. 2016;43:200-2.

19. Solé-Boldo L, Raddatz G, Schütz S, et al. Single-cell transcriptomes of the human skin reveal age-related loss of fibroblast priming. Commun Biol. 2020;3: 188.

20. Zuo Z, Wang B, Shen M, et al. Skincare habits and rosacea in 3,439 Chinese adolescents: a universitybased cross-sectional study. Acta Derm Venereol. 2020;100: adv00081.

21. Li J, Wang B, Deng Y, et al. Epidemiological features of rosacea in Changsha, China: a population-based, cross-sectional study. J Dermatol. 2020;47:497-502.

22. Zouboulis CC, Chen WC, Thornton MJ, Qin K, Rosenfield R. Sexual hormones in human skin. Hormone and metabolic research Hormon- und Stoffwechselforschung Hormones et metabolisme. 2007;39:85-95.

23. Del Rosso JQ. Advances in understanding and managing rosacea: part 1: connecting the dots between pathophysiological mechanisms and common clinical features of rosacea with emphasis on vascular changes and facial erythema. J Clin Aesthet Dermatol. 2012;5:16-25.

24. Hirt PA, Castillo DE, Yosipovitch G, Keri JE. Skin changes in the obese patient. J Am Acad Dermatol. 2019;81:1037-57.

25. Seo YJ, Li ZJ, Choi DK, et al. Regional difference in sebum production by androgen susceptibility in human facial skin. Exp Dermatol. 2014;23:70-2.

26. Smith KE, Fenske NA. Cutaneous manifestations of alcohol abuse. J Am Acad Dermatol. 2000;43:1-16 (Quiz -8).

27. Higgins E, du Vivier A. Alcohol intake and other skin disorders. Clin Dermatol. 1999;17:437-41.

28. Li S, Cho E, Drucker AM, Qureshi AA, Li WQ. Alcohol intake and risk of rosacea in US women. J Am Acad Dermatol. 2017;76:1061-7.e2.

29. Curnier A, Choudhary S. Rhinophyma: dispelling the myths. Plast Reconstr Surg. 2004;114:351-4. 